\title{
Dextrin nanoparticles: Studies on the interaction with murine macrophages and blood clearance
}

\author{
Catarina Gonçalves $^{\mathrm{a}}$, Egídio Torrado ${ }^{\mathrm{b}}$, Teresa Martins ${ }^{\mathrm{b}}$, Paula Pereira ${ }^{\mathrm{a}}$, Jorge Pedrosab ${ }^{\mathrm{b}}$, Miguel Gama ${ }^{\mathrm{a}, *}$ \\ a IBB-Institute for Biotechnology and Bioengineering, Centre for Biological Engineering, Minho University, Campus de Gualtar, 4710-057 Braga, Portugal \\ ${ }^{\mathrm{b}}$ Life and Health Sciences Research Institute (ICVS), School of Health Sciences, Braga, Portugal
}

\section{A R T I C L E I N F O}

\section{Article history:}

Received 3 July 2009

Received in revised form

15 September 2009

Accepted 19 September 2009

Available online 25 September 2009

\section{Keywords:}

Nanoparticles

Macrophages

Fluorescence

Cellular uptake

Fluorescein isothiocyanate (FITC)

\begin{abstract}
A B S T R A C T
The uptake of nanoparticles by cells of the mononuclear phagocytic system limits its use as colloidal drug carriers, reducing the blood circulation time and the ability to reach biological targets. In this work, the interaction between dextrin nanoparticles - recently developed in our laboratory - and murine bone marrow-derived macrophages was evaluated. Cytotoxicity and nitric oxide production were studied, using the MTT assay and the Griess method, respectively. FITC labelled nanoparticles were used to assess the phagocytic uptake and blood clearance after intravenous injection. The phagocytic uptake was analysed in vitro by confocal laser scanning microscopy and fluorescence activated cell sorting. The results show that the nanoparticles are not cytotoxic and do not stimulate the production of nitric oxide by macrophages, in the range of concentrations studied. Nanoparticles are phagocytosed by macrophages and are detected inside the cells, concentrated in cellular organelles. The blood clearance study showed that the blood removal of the nanoparticles occurs with a more pronounced rate in the first $3 \mathrm{~h}$ after intravenous administration, with about $30 \%$ of the material remaining in systemic circulation at this stage. Given the fairly high blood circulation time and biocompatibility, the dextrin nanoparticles are promising carriers for biomedical applications. Both applications targeting phagocytic, antigen-presenting cells (for vaccination purposes) and different tissues (as drug carriers) may be envisaged, by modulation of the surface properties.
\end{abstract}

(C) 2009 Elsevier B.V. All rights reserved.

\section{Introduction}

Many colloidal carriers, such as liposomes, nanocapsules and nanoparticles, have been developed as intravenous drug delivery systems. These systems may improve the absorption of poorly water-soluble pharmaceuticals, protect sensitive molecules against in vivo degradation, modify the distribution of drugs in the body and increase the patient's compliance, by avoiding repeated injections. The rapid removal of intravenously administered colloidal drug carrier systems by the mononuclear phagocytic system (MPS) is an obstacle to the efficient targeting of solid tumours and inflammatory tissues [1,2]. When nanoparticles enter the bloodstream, they instantly encounter a complex environment of plasma proteins and phagocytic cells. Opsonization promotes recognition and uptake by cells of the MPS, especially macrophages in the liver (the Kupffer cells), but also spleen and bone marrow macrophages [3]. Recent research has focused on the modification of nanoparticles surface to avoid opsonization. In this

\footnotetext{
* Corresponding author.

E-mail address: fmgama@deb.uminho.pt (M. Gama).
}

context, poly(ethylene glycol) (PEG) was found to decrease the interactions of the particles with blood proteins, avoiding recognition by the MPS and thus increasing the circulation time in the blood [4]. As one of the most promising alternatives to PEG, polysaccharides have been widely investigated. The advantage of polysaccharide coating is the steric protection of nanoparticles against non-specific interactions with proteins, ensuring particle stability in blood circulation. Additionally, as polysaccharides offer reactive groups for modification, active targeting may be obtained by grafting ligands onto the nanoparticles surface [5-8].

Knowledge on the interaction between macrophages and drug carriers is essential in the design of more effective therapeutic strategies using nanobiotechnological devices. Macrophages may be the therapeutic target, for the treatment of macrophageassociated pathologies or vaccination purposes, given their activity as antigen-presenting cells [9,10]. Otherwise, when other targets are envisaged, phagocytic activity should ideally be avoided, providing the drug carrier time enough to reach the target. Macrophages are phagocytic cells that produce antimicrobial molecules, in the phagosome, and secrete a variety of substances, whose actions range from induction of cell growth to 
cell death, in addition to their role in antigen-presentation to $\mathrm{T}$ cells. These substances produced by macrophages, such as nitric oxide and cytokines, are involved in host defence against pathogenic micro-organisms, parasites or tumour cells. The phagocytic process starts when macrophages recognize foreign particles or pathogens, becoming thereafter activated, a process that leads to the secretion of a number of chemical mediators of inflammation. These molecules are very aggressive, not only against foreign molecules or particles, but also to the host tissues. Among them, nitric oxide (NO) is a key marker of activation of inflammation [11].

After administration of colloidal carriers, the in vivo distribution largely depends on their particle size [12] and surface properties [13]. As referred above, in many instances it is desirable to increase the blood circulation time of the drug carriers. Elimination of the nanoparticles from systemic circulation can occur by different mechanisms: uptake by phagocytic cells, extravasation through endothelium and renal excretion. Nanoparticle uptake by the phagocytic cells may occur both in the bloodstream by monocytes and in tissues by resident phagocytes (e.g., Kupffer cells in the liver, dendritic cells in the lymph nodes, spleen macrophages). Nanoparticles can escape the circulation through the gaps, also called fenestrations, of the endothelial barrier. Fenestrations and the vasculature can undergo modifications under various pathological conditions. For instance, tumor growth induces the development of neovasculature characterized by discontinuous endothelium, with large fenestrations. Depending on the reports, the "ideal" size requirements for nanoparticles developed for cancer treatment vary from 70 to $200 \mathrm{~nm}$. Larger particles can be rapidly taken up by the mononuclear phagocytic system cells [14]. Small particles $(<5.5 \mathrm{~nm})$ are described to be rapidly and efficiently excreted in the urine [15].

In previous work, nanoparticles obtained by self-assembling of hydrophobized dextrin were developed and characterized in our laboratory. The nanoparticles obtained have high colloidal stability and spherical shape [16]. Size distribution obtained by dynamic light scattering showed two distinct populations, with 25 and $150 \mathrm{~nm}$, the former being the predominant one [17]. In the present work, the ability of these nanoparticles to interact with bone marrow-derived macrophages (BMDMs) was analysed. The use of nanoparticles labelled with a fluorochromic probe allowed the detection of internalization by BMDM and the evaluation of the blood clearance profile. These are the major goals of this work: to ascertain whether the dextrin nanoparticles are effectively internalized by phagocytic cells (relevant for vaccination purposes) and estimate the blood circulation time (crucial for the development of drug delivery applications).

\section{Materials and methods}

\subsection{Materials}

Dextrin-VA-SC $\mathrm{S}_{16}$ (VA: vinyl acrylate, $\mathrm{SC}_{16}$ : alkyl chain) was synthesized as comprehensively described previously [16]. In this work, a sample of dextrin-VA-SC ${ }_{16}$ (dexC $C_{16}$ ) with 13 acrylate groups $\left(\mathrm{DS}_{\mathrm{VA}} 13 \%\right)$ and 6 alkyl chains $\left(\mathrm{DS}_{\mathrm{C} 16} 6 \%\right)$ per 100 dextrin glucopyranoside residues was used. Dimethylsulfoxide was purchased from Fluka. The chemicals $4^{\prime}, 6-$ diamidino-2-phenylindole (DAPI), lipopolysaccharide (LPS), saponine and 3-(4,5-dimethylthiazol-2-yl)-2,5-diphenyltetrazolium bromide (MTT) were purchased from Sigma-Aldrich. SAMSA (5(2-(and-3)-S-(acetylmercapto)succinoyl) amino) fluorescein was obtained from Invitrogen. IFN- $\gamma$ was obtained from R\&D systems. Sephadex G25 PD10 columns were obtained from Amersham Biosciences.

\subsection{Animals}

Eight-week-old female BALB/c mice, an inbred strain with high genetic homogeneity between individuals, obtained from Harlan Interfauna Ibérica (Barcelon, Spain), were housed under specificpathogen-free conditions with food and water ad libitum. The experiments involving mice were conducted under the guidelines and approval of the Research Ethics Committee of the Life and Health Sciences Research Institute (Braga, Portugal) and of the governmental agency Direç̧ão Geral de Veterinária (Lisbon, Portugal).

\subsection{Culture of murine BMDM}

Macrophages were obtained from the bone marrow, as previously described $[18,19]$. This method allows the differentiation of a homogenous primary culture of macrophages that retains the morphological, physiological and surface marker characteristics of these phagocytes [20,21,22]. Briefly, mice were sacrificed using $\mathrm{CO}_{2}$, and the femurs were removed under aseptic conditions. Bones were flushed with $5 \mathrm{~mL}$ cold Hanks' balanced salt solution (HBSS; Gibco, Paisley, United Kingdom). The resulting cell suspension was centrifuged at $500 \times g$ and resuspended in Dulbecco's modified Eagle's medium (DMEM; Gibco), supplemented with $10 \mathrm{mM}$ HEPES (Sigma, St. Louis, MO), $1 \mathrm{mM}$ sodium pyruvate (Gibco), $10 \mathrm{mM}$ glutamine (Gibco), 10\% heat-inactivated fetal bovine serum (Sigma), and 10\% L929 cell conditioned medium (complete DMEM [cDMEM]). To remove fibroblasts or differentiated macrophages, cells were cultured for a period of $4 \mathrm{~h}$ on cell culture dishes (Nunc, Naperville, IL) with cDMEM. Nonadherent cells were collected with warm HBSS, centrifuged at $500 \times \mathrm{g}$, distributed in 24-well plates at a density of $5 \times 10^{5}$ cells/well, and incubated at $37^{\circ} \mathrm{C}$ in a $5 \% \mathrm{CO}_{2}$ atmosphere. On day 4 after seeding, $0.1 \mathrm{~mL}$ of $\mathrm{L} 929$ cell conditioned medium was added, and medium was renewed on the seventh day. After 10 days in culture, cells were completely differentiated into macrophages.

\subsection{Preparation of self-assembled nanoparticles}

Lyophilized dex $\mathrm{C}_{16}$ was dissolved in culture medium under stirring and further sonicated for $20 \mathrm{~min}$. A stock solution $(3 \mathrm{mg} / \mathrm{mL})$ of nanoparticles was prepared. The nanoparticle concentration was adjusted by dilution of concentrated nanoparticle dispersion, with culture medium, in the wells. All solutions were sterilized by filtration through a $0.22 \mu \mathrm{m}$ membrane. The nanoparticles formation was confirmed by dynamic light scattering and visualized in atomic force microscopy.

The size distribution was determined with a Malvern Zetasizer, MODEL NANO ZS (Malvern Instruments Limited, UK). A dispersion of nanoparticles was analysed at $25^{\circ} \mathrm{C}$ in a polystyrene cell, using a He-Ne laser-wavelength of $633 \mathrm{~nm}$ and a detector angle of $173^{\circ}$.

Tapping mode imaging was carried out on a Nanoscope IIIa Multimode (Digital Instruments, Veeco) scanning probe microscope. A silicon tip doped with phosphorus, with a radius curvature of less than $10 \mathrm{~nm}$ (RTESP, VEECO), was used. This tip has a typical resonance frequency of $288-328 \mathrm{kHz}$ and a typical force constant of $20-80 \mathrm{~N} / \mathrm{m}$. A scan rate of $1.4-1.8 \mathrm{~Hz}$ was sufficient to maintain a good signal-to-noise ratio. A drop of nanoparticles dispersion was placed on new-cleaved HOPG surface, thoroughly rinsed with water and dried under a $\mathrm{N}_{2}$ flux.

\subsection{Cytotoxicity test}

The cytotoxicity of the dextrin nanoparticles was evaluated, in vitro, using the 3-(4,5-dimethylthiazol-2-yl)-2,5-diphenyltetrazolium bromide (MTT) assay. The tetrazolium salt is widely used to quantify cytotoxicity, by colorimetry. The tetrazolium salts 
are metabolically reduced to highly coloured end products, formazans [23]. The colourless MTT is cleaved to formazan by the succinate-tetrazolium reductase system which belongs to the mitochondrial respiratory chain and is active only in viable cells.

Nanoparticles, at different concentrations, were incubated with cells, for 24 or $48 \mathrm{~h}$. Then, MTT was added to the culture medium to a final concentration of $0.5 \mathrm{mg} / \mathrm{mL}$. After $3 \mathrm{~h}$ incubation, the insoluble formazan crystals were solubilized with DMSO, and the absorption was measured at 570 and $690 \mathrm{~nm}$ in an automated ELISA plate reader. For each sample, the background optical density $(690 \mathrm{~nm})$ was subtracted; the test was performed in triplicate.

\subsection{Nitric oxide quantification}

The nitric oxide production was evaluated by quantifying the nitrite accumulation in cell culture supernatants using the Griess method [24]. Despite this method quantifies only nitrites, not nitrates, for the sake of the comparison carried out in this work it is sufficient. As a positive control, BMDM culture was challenged with lipopolysaccharide (LPS) and gamma interferon (IFN- $\gamma$ ). LPS is an endotoxin of the bacterial cell wall, well known to stimulate the release of different markers of inflammation by macrophage cells. It is used to stimulate macrophages in order to obtain a background of activation/inflammation, as it is usually done in studies concerning the inflammatory potential of different biochemical agents. IFN- $\gamma$ is a cytokine produced by T-lymphocytes and NK cells which activates the antimicrobial mechanism of macrophages.

BMDM cells $\left(5 \times 10^{5}\right.$ cells/well $)$ were incubated, at $37^{\circ} \mathrm{C}$ in a $5 \%$ $\mathrm{CO}_{2}$ atmosphere, for 24 or $48 \mathrm{~h}$, in the presence of different concentrations of nanoparticles $(1.0,0.5$ and $0.1 \mathrm{mg} / \mathrm{mL})$. Assays were carried out either with and without LPS $(100 \mathrm{ng} / \mathrm{mL})$ and IFN- $\gamma$ $(1 \mathrm{ng} / \mathrm{mL})$. The final volume used was $1 \mathrm{~mL}$ per well. Then, $100 \mu \mathrm{L}$ of Griess reagent was added to $100 \mu \mathrm{L}$ of sample culture medium, and the absorbance was measured at $550 \mathrm{~nm}$. Nitrite concentrations in the medium were finally determined by using standard solutions of sodium nitrite $(0-100 \mu \mathrm{M})$.

\subsection{Uptake of nanoparticles by the BMDM}

\subsubsection{Preparation of FITC labelled nanoparticles}

FITC is a fluorescent probe quite commonly used in biological studies, owing to its biocompatibility. In order to label the nanoparticles with FITC, the following solutions were prepared: (1) Nanoparticle solution-10 mg of dexC $C_{16}$ were dissolved in $1.3 \mathrm{~mL}$ of sodium phosphate buffer $0.1 \mathrm{M} \mathrm{pH} 7$ and stirred for $30 \mathrm{~min}$; (2) fluorescein solution $-5 \mathrm{mg}$ of SAMSA fluorescein were dissolved in $0.5 \mathrm{~mL}$ of $\mathrm{NaOH} 0.1 \mathrm{M}$ and stirred for $15 \mathrm{~min}$. Afterwards, $7 \mu \mathrm{L}$ of $\mathrm{HCl} 6 \mathrm{M}$ and $0.1 \mathrm{~mL}$ of $\mathrm{NaPO}_{4}$ buffer $0.5 \mathrm{M} \mathrm{pH} 7$ were added and stirred for $10 \mathrm{~min}$. Finally, these two solutions were mixed up and stirred for $30 \mathrm{~min}$. Unbound FITC was separated using a Sephadex G25 PD10 column equilibrated with PBS. Nanoparticles labelled with FITC were eluted with PBS and sterilized by filtration through a $0.22 \mu \mathrm{m}$ membrane before use. FITC labelling did not affect the properties of the nanoparticles, as estimated by dynamic light scattering.

\subsubsection{Fluorescence studies}

The assessment of phagocytosis, after incubating the fluorescent-labelled nanoparticles with murine macrophages, was attempted by spectrofluorimetry. In this study, FITC-containing nanoparticles $(1.0,0.5$ or $0.1 \mathrm{mg} / \mathrm{mL})$ were added to BMDM cultures, which were pre-stimulated with LPS $(100 \mathrm{ng} / \mathrm{mL})$ and IFN- $\gamma$ $(1 \mathrm{ng} / \mathrm{mL}$ ) on a 96 -well plate. As a control, $0.5 \mathrm{mg} / \mathrm{mL}$ nanoparticles were incubated with cells without LPS and IFN- $\gamma$. Following incubation periods of 3 or $6 \mathrm{~h}$, the culture medium was collected and cell monolayers were rinsed four times with PBS to remove the non-phagocytosed nanoparticles. Then, $200 \mu \mathrm{L}$ culture medium and $2 \mu \mathrm{L}$ saponine (10\%) were added and the cell lysates collected $10 \mathrm{~min}$ after incubation. Fluorescence intensity was measured in both the cell lysate and the collected culture medium, using a spectrofluorimeter.

\subsubsection{Confocal observation of the macrophages}

In order to evaluate the phagocytic activity, macrophages $\left(5 \times 10^{5}\right.$ cells/well) were seeded on coverslips and stimulated with LPS $(100 \mathrm{ng} / \mathrm{mL})$ and IFN- $\gamma(1 \mathrm{ng} / \mathrm{mL})$. Then, the cell culture was incubated with or without FITC-containing nanoparticles (1.0 or $0.1 \mathrm{mg} / \mathrm{mL}$ ) for $6 \mathrm{~h}$. The cover glasses were washed twice with PBS and cells were fixed with methanol absolute $\left(-20^{\circ} \mathrm{C}\right)$ for $10 \mathrm{~min}$. Following PBS washing (twice), cells were labelled with DAPI (staining nucleous-blue) for $15 \mathrm{~min}$. Vectashield was used as an anti-fading. Cover glasses were analysed using a laser scanning confocal microscope LEICA SP AOBS SE (Leica Microsystems, Germany), equipped with HC PL APO Lbl. Blue 63x/140 Oil objective.

\subsubsection{FACS analysis}

Cells $\left(5 \times 10^{5}\right.$ cells $\left./ \mathrm{mL}\right)$ were seeded in 96 -well plates and incubated at $37{ }^{\circ} \mathrm{C}$ and $5 \% \mathrm{CO}_{2}$. After removal of the growth medium, the cells were pre-stimulated with LPS $(100 \mathrm{ng} / \mathrm{mL})$ and IFN- $\gamma$ $(1 \mathrm{ng} / \mathrm{mL})$ and treated with FITC-linked nanoparticles $(1.0,0.5$ or $0.1 \mathrm{mg} / \mathrm{mL}$ ), for 3 or $6 \mathrm{~h}$. As a control, $0.5 \mathrm{mg} / \mathrm{mL}$ nanoparticles were incubated with cells not pre-stimulated with LPS and IFN- $\gamma$. After incubation, cells were washed four times with PBS and collected with $100 \mu \mathrm{L}$ of cooled EDTA. Then cells were transferred to FACStubes with $1 \mathrm{~mL}$ PBS and centrifuged at $500 \times g$ for $10 \mathrm{~min}$. After removal of the supernatant, cells were resuspended in $500 \mu \mathrm{L}$ of FACS buffer (EDTA with 1\% BSA).

Flow cytometric analysis was performed using a FACS Coulter Epics XL (Applied Cytometry Systems, United Kingdom). The cells were excited with a $488 \mathrm{~nm}$ laser and green fluorescence collected using a $530 \pm 30 \mathrm{~nm}$ band pass filter. Data were analysed using EXPO32 software (Applied Cytometry Systems, United Kingdom), which statistical functions were used to quantify the proportion of cells with green fluorescence, corresponding to the percentage of macrophages with phagocytosed nanoparticles.

\subsubsection{Blood clearance}

On the day of experiments, mice were grouped $(N=3)$ according to pre-defined periods of time $(0,3,5,10$ and $24 \mathrm{~h})$. A solution of NPsFITC $(100 \mu \mathrm{L}, 0.3 \mathrm{mg} / \mathrm{mL})$ was injected in the tail vein. The injections were well tolerated and no adverse effects were recorded during the observation period. After pre-defined periods following nanoparticles administration, blood was collected through tail cut. Blood was centrifuged $\left(14,000 \mathrm{rpm}, 40 \mathrm{~min}, 4^{\circ} \mathrm{C}\right)$ and the fluorescence of supernatant was analysed. The samples corresponding to the beginning of the experiment $(0 \mathrm{~h})$ were collected immediately after injection.

Fluorescence measurements were performed on a VARIAN Cary Eclipse fluorescence spectrofluorometer using a quartz cell. The FITC spectra were obtained using an excitation wavelength of $485 \mathrm{~nm}$, and recording the emission over the range 500-590 nm, at a scan rate of $125 \mathrm{~nm} \mathrm{~min}^{-1}$. The slit width was set at $5 \mathrm{~nm}$ for the excitation and $10 \mathrm{~nm}$ for the emission.

\subsubsection{Results and discussion}

Self-assembled nanoparticles were observed using dynamic light scattering (DLS) and atomic force microscopy after filtration through a $0.22 \mu \mathrm{m}$ membrane. The AFM image (Fig. 1a) shows that nanoparticles have spherical shape. The fundamental size distribution generated by DLS is an intensity distribution - quite sensitive 

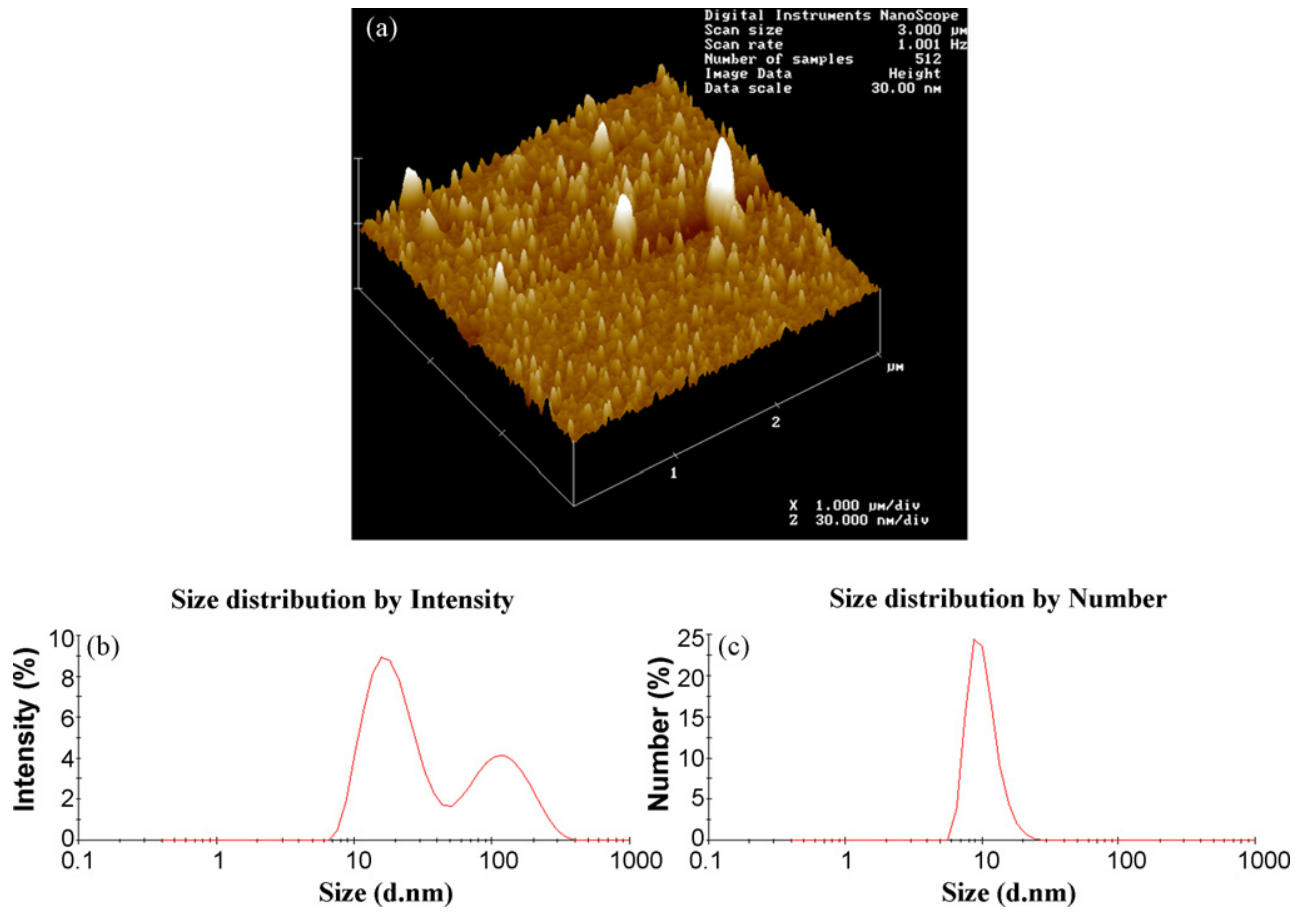

Fig. 1. AFM image (a) and size distribution by intensity (b) and by number (c) of nanoparticles dispersion (1 mg/mL).

to the presence of larger particles - which can be converted, using Mie theory, to a volume distribution. This volume distribution can also be further converted to a number distribution. The DLS analysis reveal two populations in the intensity distribution (Fig. 1b), with roughly 20 and $100 \mathrm{~nm}$. The conversion to number distribution (Fig. 1c) highlights only the smaller population of nanoparticles, the predominant one. The nanoparticles size was not affected by the FITC labelling (data not shown).

It should be remarked that the material used on the development of the nanoparticles, dextrin, is a very promising biomaterial, available in medical grade and accepted by FDA [25,26]. However, in spite of its biocompatibility and availability on large amounts, it is under exploited in the biomedical field.

\subsubsection{BMDM cultures: cytotoxicity and nitric oxide production}

The nanoparticles toxicity was studied using BMDM cultures and the MTT assay. Fig. 2a shows the results. The presence of nanoparticles, in the range of concentrations studied, does not affect cell viability as measured by the MTT assay. Cells treated with nanoparticles showed a MTT optical density similar to the control (untreated cells). Furthermore, the cells exhibit normal morphology. Similar experiments performed with fibroblast cultures produced similar results (data not shown). It must be remarked that the concentration of nanoparticles used in these assays exceeds the values likely to be reached in vivo, therefore it may be concluded that the dextrin nanoparticles are not cytotoxic.

The nanoparticles effect on the nitric oxide production by cultured macrophages was analysed (Fig. 2b). Cells were incubated for $48 \mathrm{~h}$ with nanoparticles alone and the production of nitric oxide was not detected in the conditions of the assay. Moreover, as expected, the addition of LPS and IFN- $\gamma$ to the culture medium induced nitric oxide production. The effect of combining LPS and IFN- $\gamma$ with different nanoparticles concentration was therefore studied. This analysis revealed that the release of nitric oxide was similar, irrespective of the presence of nanoparticles. The presence of the nanoparticles seems not to promote any inflammatory action or elicit a reactive response when in contact with macrophages.
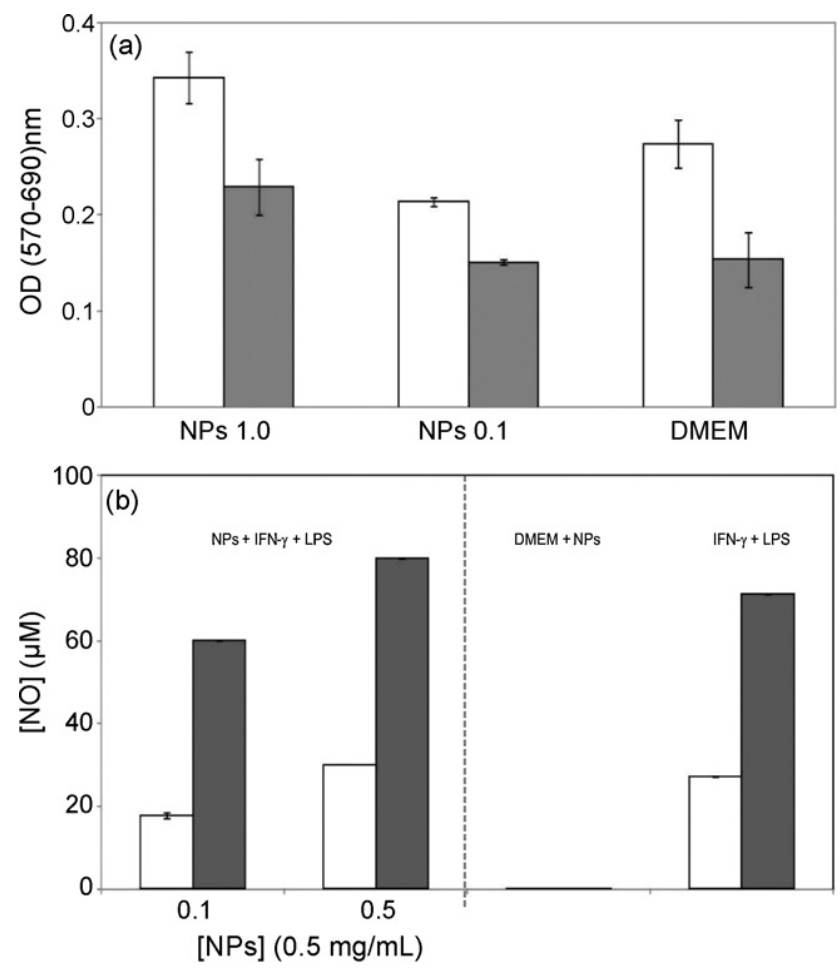

Fig. 2. BMDM viability (a) with different nanoparticles concentrations (1.0 or $0.1 \mathrm{mg} / \mathrm{mL}$ ) and nitric oxide production (b) using 0.5 or $0.1 \mathrm{mg} / \mathrm{mL}$ nanoparticles, after $24 \mathrm{~h}(\square)$ or $48 \mathrm{~h}(\square)$ of incubation. For nitric oxide assay BMDM were stimulated with LPS $(100 \mathrm{ng} / \mathrm{mL})$ and IFN- $\gamma(1 \mathrm{ng} / \mathrm{mL})$. For negative control cells were incubated only with nanoparticles. The error bar corresponds to the standard deviation.

\subsubsection{Uptake of nanoparticles}

Particle size is known to play a primary role in the interaction of nanoparticles with phagocytic cells. Larger particles are rapidly taken up by the mononuclear phagocytic system, and smaller ones have a minor chance of being sequestered by phagocytic cells. 

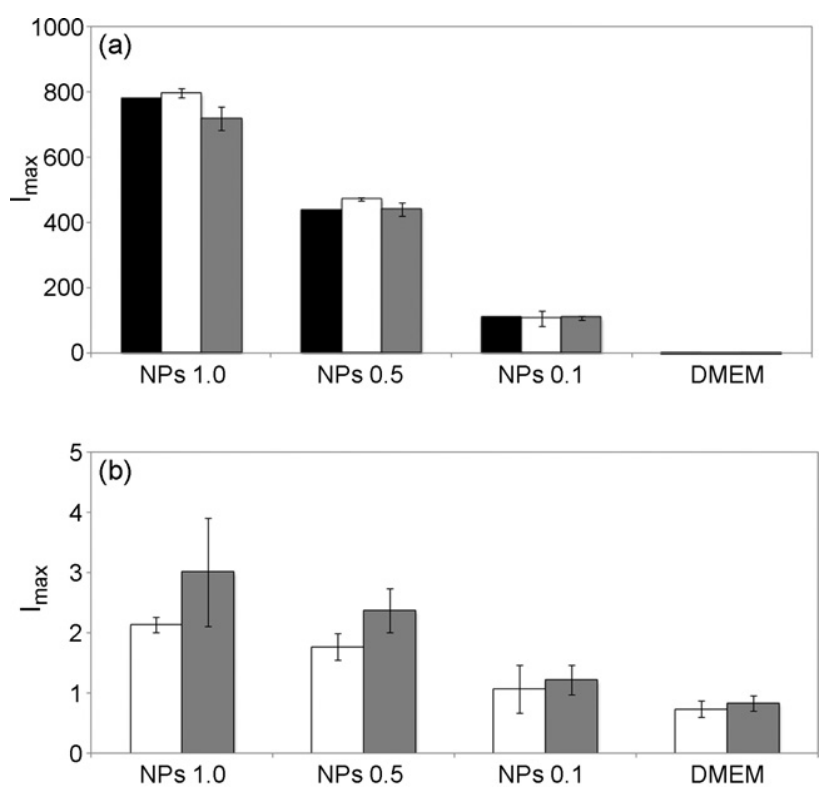

Fig. 3. Fluorescence intensity of the FITC labelled nanoparticles in the culture medium before $(t=0 \mathrm{~h})$ and after $(t=3$ or $6 \mathrm{~h})$ contact with cells (a) and for BMDM cell lysate (b) after incubation with $1.0,0.5$ or $0.1 \mathrm{mg} / \mathrm{mL}$ nanoparticles. Bars represent $0 \mathrm{~h}(\square), 3 \mathrm{~h}(\square)$ or $6 \mathrm{~h}(\square)$. The error bar corresponds to the standard deviation.

Therefore, the size of the nanoparticles has a critical effect on their behavior in biological systems.

In order to detect the internalization of the nanoparticles by the macrophages, fluorescence intensity was measured before $(0 \mathrm{~h})$ and after 3 or $6 \mathrm{~h}$ of contact of FITC labelled nanoparticles with BMDM, both in cell lysates and in the culture medium. The results are shown in Fig. 3.

The fluorescence intensity was assessed in the macrophage culture medium, following incubation with the nanoparticles. No differences were seen throughout the periods of observation (Fig. 3a), the majority of nanoparticles remaining outside the phagocytic cells.

However, when lysates of BMDM cultivated in the presence of nanoparticles were analysed, an increased fluorescence intensity (Fig. 3b) was detected at the higher concentrations tested. The variations in fluorescence intensity, although slight ones, suggest the occurrence of phagocytic processes. The hardly detectable variations in fluorescence intensity suggest that the nanoparticles concentration is far beyond the saturation of the cells. The cell activation does not affect the phagocytic activity of BMDM, since no significant difference was observed irrespective of the stimulation with LPS and IFN- $\gamma$ (data not shown).

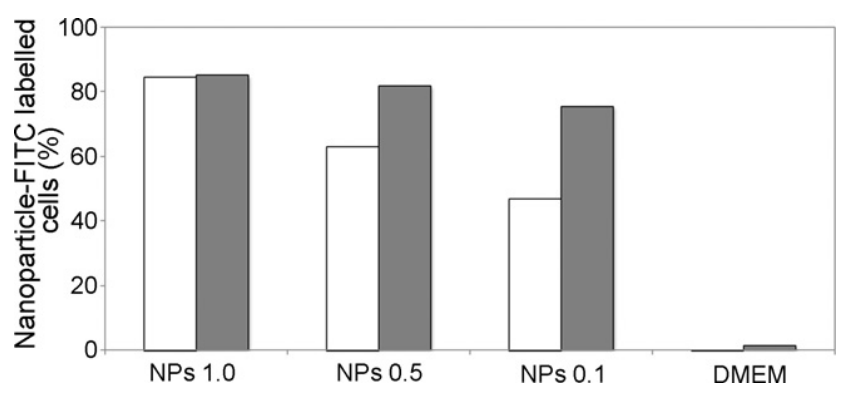

Fig. 5. Percentage of macrophage cells that incorporated FITC-labelled nanoparticles $(1.0,0.5$ and $0.1 \mathrm{mg} / \mathrm{mL})$, after $3 \mathrm{~h}(\square)$ or $6 \mathrm{~h}(\square)$, measured by FACS analysis.

Bocca et al., using the same methodology, reported substantial differences in the internalization of stealth and non-stealth solid lipid nanoparticles (average diameter $100 \mathrm{~nm}$ ), the former undergoing the fastest uptake. The phagocytic uptake of stealth fluorescent nanoparticles was very low - as in this work, with the dextrin nanoparticles - corresponding to about $5 \%$ of the initial dose, after $90 \mathrm{~min}$ of incubation with murine macrophages [27]. Fang et al. reported the influence of nanoparticles size on macrophage uptake. The study was performed using poly methoxypolyethyleneglycol cyanoacrylate-co-n-hexadecyl cyanoacrylate (PEG-PHDCA) nanoparticles with different size (80, 170 and $240 \mathrm{~nm}$ ). No significant uptake differences were observed in the first $0.5 \mathrm{~h}$. However, after $2 \mathrm{~h}$ remarkable differences were observed and the uptake percentage was lower for nanoparticles with $80 \mathrm{~nm}$ [28]. Again, as in this work, only a minor fraction of the nanoparticles are taken up by the cells.

Confocal microscopy was used to further examine the uptake of FITC labelled nanoparticles by macrophages. In the control culture (Fig. 4a) only nucleus stained with DAPI are visible. Cells incubated with nanoparticles show small green spots (Fig. 4b and c), in the cytoplasm, that can be attributed to the FITC in the internalised nanoparticles. Fluorescent material is concentrated in cellular organelles, irrespective of the concentration used. The cell membrane is not visible, suggesting that the nanoparticles are not adsorbed on the cell surface, as confirmed by the FACS analysis (see below). Apparently, as suggested by the fluorescence depletion studies, the nanoparticles concentration seems to largely exceed the uptake ability of macrophages. Ongoing work focuses on the analysis of the nanoparticles intracellular trafficking.

The results obtained by FACS analysis confirm the confocal microscopy observations. The cell viability was confirmed with propidium iodide. The percentage of fluorescent macrophages after incubation with labelled nanoparticles was very high, as shown in Fig. 5. Furthermore, an additional assay using a cell-impermeable dye (trypan blue, a FITC quencher), was performed. Since the fluorescence was not reduced in the presence of trypan blue
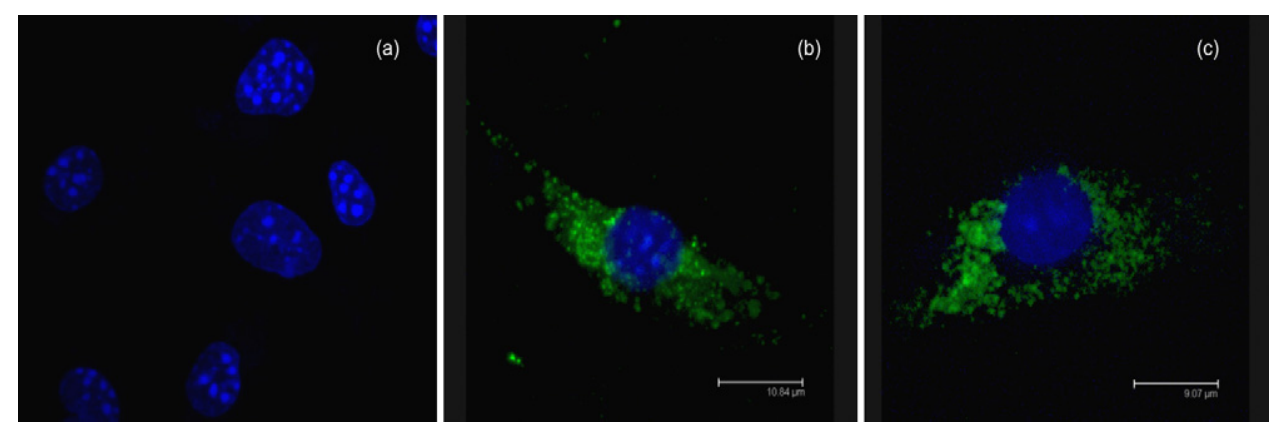

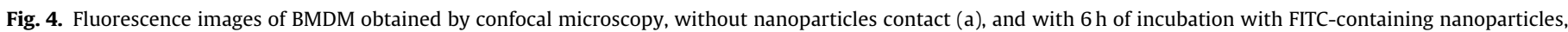
$0.1 \mathrm{mg} / \mathrm{mL}$ (b) or $1.0 \mathrm{mg} / \mathrm{mL}$ (c). 
(data not shown), it may be concluded that the nanoparticles are indeed concentrated inside the macrophages, as shown by confocal microscopy, and not just adsorbed on the cell membrane.

As suggested by the confocal microscopy images, shown in Fig. 4, the amount of nanoparticles internalised is similar for the different concentrations used, suggesting that a saturation of the uptake ability of the cells occur in the range of concentrations tested. The phagocytic activity of BMDM cells is not affected by activation with LPS and IFN- $\gamma$, since no significant difference was observed (data not shown). The effective internalization suggests that the nanoparticles may be used for vaccination purposes, carrying synthetic antigens to phagocytic cells. Considering this possibility, ongoing work aims at clarifying the kinetics of the phagocytosis, the concentration dependence effects, and the intracellular fate of the nanoparticles.

\subsubsection{Blood clearance}

Long systemic circulation time is a highly demanded feature for nanoparticles-based drug delivery systems, providing the carriers enough time to reach their targets and to release their content in a continuous and controlled-way.

The FITC labelled nanoparticles were used for blood clearance studies, after intravenous injection. The blood clearance profile for FITC labelled nanoparticles is shown in Fig. 6. The elimination of nanoparticles from systemic circulation is relatively fast in the first $3 \mathrm{~h}$, then proceeding at lower rate. In the first period studied, $3 \mathrm{~h}$, about $65 \%$ of the nanoparticles were removed from the bloodstream and in the next $2 \mathrm{~h}$ another $15 \%$ fade away. At $24 \mathrm{~h}$, only $5 \%$ of the injected dose is circulating in the bloodstream. The clearance rate of dextrin nanoparticles observed in the blood clearance profile may be related with the nanoparticles size distribution. The small nanoparticles should be small enough to cross the endothelial barrier or to be eliminated by renal clearance. Thus, the nanoparticles remaining for longer periods may correspond to the population of larger nanoparticles. Further biodistribution studies by scintigraphy are being carried out in our laboratory, using nanoparticles labeled with radioactive samarium (complexed with DOTA). Preliminary results show renal accumulation of the nanoparticles and consequent excretion in the urine, thus reinforcing the hypothesis of filtration of the smallest nanoparticles, at least in the kidney. Biodegradation by serum $\alpha$-amylase is not likely to occur. Indeed, enzymatic hydrolysis of $\operatorname{dex}_{16}$ by $\alpha$-amylase, in vitro, was not detected by dynamic light scattering or reducing sugar method (data not shown). We assume that the polymer modification with alkyl chains blocks the enzyme action: since the dextrin used has very low degree of polymerization - about 12 - every glucose residue is quite close to the alkylic graft, which is likely to avoid the amylase action.

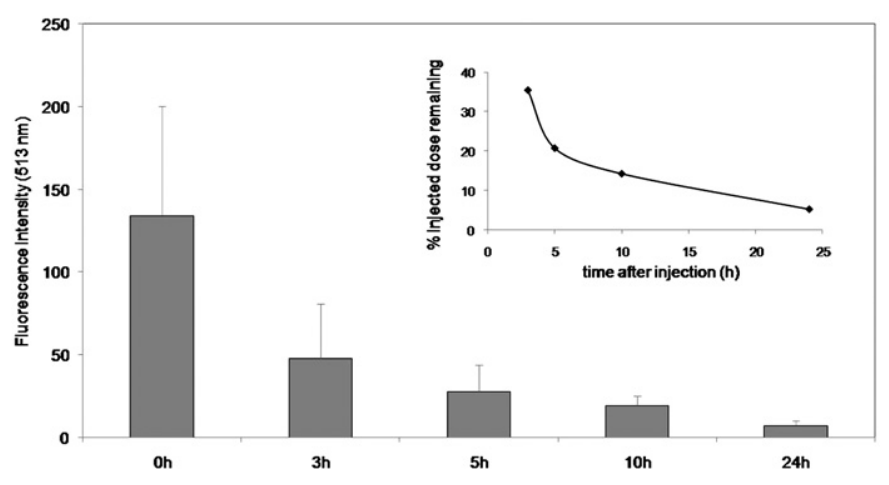

Fig. 6. Blood clearance profile of FITC-labelled nanoparticles. Inset shows the percentage of the initial $(0 \mathrm{~h})$ fluorescence, remaining in the bloodstream at different time. The error bar corresponds to the standard deviation.
The blood clearance profile obtained is very similar to others reported for polymeric nanoparticles. Micelles prepared from conjugates of polyethylene glycol (PEG) and phosphatidylethanolamine (PE), with the size of $7-35 \mathrm{~nm}$, have circulation half-lives from 1.2 to $2.1 \mathrm{~h}$ depending on the molecular size of PEG block [29]. A recent study evaluated the circulation kinetics and biodistribution of core-crosslinked (CCL) and non-crosslinked (NCL) micelles based on $\mathrm{mPEG}_{5000}$ and $N$-(2-hydroxyethyl)methacrylamide-oligolactates (mPEG- $b$ $\left.\mathrm{p}\left(\mathrm{HEMAm}-\mathrm{Lac}_{\mathrm{n}}\right)\right)$. CCL and NCL micelles have the same size $(57 \mathrm{~nm})$. The NCL micelles were rapidly eliminated from the circulation and only $6 \%$ of the injected dose was present after $4 \mathrm{~h}$, probably due to interactions with plasma proteins which lead to recognition by macrophages. Contrary to NCL assemblies, CCL micelles displayed significantly prolonged circulation times, and $50 \%$ of the injected dose was still present in the systemic circulation after $6 \mathrm{~h}$. At $24 \mathrm{~h}$ post-injection, $5 \%$ of the injected dose was in the blood circulation and $10 \%$ and $1.7 \%$ were taken up by the liver and spleen, respectively. A relatively high accumulation in the abdominal skin was observed, attributed to extravasation into areas that experience pressure and/or micro-traumatised areas in the skin, which is allowed by long circulation time and small size [30].

\section{Conclusions}

In vitro studies with BMDM revealed that nanoparticles are noncytotoxic and do not elicit a reactive response when in contact with macrophages. Moreover, FACS analysis and confocal observation demonstrated that BMDM internalize the nanoparticles. A saturation of the cells uptake ability is observed in the range of concentrations tested. The in vivo study showed that, after intravenous injection, a relatively fast removal of the nanoparticles occurs in the first $3 \mathrm{~h}$, then continuing slowly up to $24 \mathrm{~h}$. The blood clearance profile reveal a moderately long circulation time, compatible with drug delivery or diagnostic applications. Dextrin nanoparticles may also be used to address phagocytic cells. Ongoing work aims at modifying nanoparticles surface with PEG, intracellular trafficking analysis and scintigraphy-based biodistribution studies.

\section{Acknowledgments}

This research was supported by Fundação para a Ciência e a Tecnologia under grant SFRH/BD/22242/2005 and POCTI program. FACS analysis carried by Manuel Vilanova and Pedro Madureira, from ICBAS - Oporto University, is greatly acknowledged.

\section{References}

[1] M.O. Oyewumi, R.A. Yokel, M. Jay, T. Coakley, R.J. Mumper, Journal of Controlled Release 95 (2004) 613.

[2] V.C.F. Mosqueira, P. Legrand, J.L. Morgat, M. Vert, E. Mysiakine, R. Gref, J.P. Devissaguet, G. Barrat, Pharmaceutical Research 18 (2001) 1411.

[3] S. Stolnik, L. Illum, S.S. Davis, Advanced Drug Delivery Reviews 16 (1995) 195

[4] A.S. Zahr, C.A. Davis, M.V. Pishko, Langmuir 22 (2006) 8178.

[5] C. Lemarchand, R. Gref, C. Passirani, E. Garcion, B. Petri, R. Muller, D. Costantini, P. Couvreur, Biomaterials 27 (2006) 108.

[6] W. Ma, X. Yuan, C. Kang, T. Su, X. Yuan, P. Pu, J. Sheng, Carbohydrate Polymers $72(2008) 75$.

[7] C. Lemarchand, R. Gref, P. Couvreur, European Journal of Pharmaceutics and Biopharmaceutics 58 (2004) 327.

[8] C. Lemarchand, R. Gref, S. Lesieur, H. Hommel, B. Vacher, A. Besheer, K. Maeder P. Couvreur, Journal of Controlled Release 108 (2005) 97.

[9] J. Schroer, A.S. Rosenthal, Springer Seminars in Immunopathology 3 (1980) 247.

[10] D.M. Underhill, M. Bassetti, A. Rudensky, A. Aderem, The Journal of Experimental Medicine 190 (1999) 1909.

[11] S. Fiorito, A. Serafino, F. Andreola, P. Bernier, Carbon 44 (2006) 1100.

[12] G. Sonavane, K. Tomoda, K. Makino, Colloids and Surfaces B: Biointerfaces 66 (2008) 274.

[13] F. Esmaeili, M. Ghahremani, B. Esmaeili, M. Khoshayand, F. Atyabi, R. Dinarvand, International Journal of Pharmaceutics 349 (2008) 249. 
[14] M. Gaumet, A. Vargas, R. Gurny, F. Delie, European Journal of Pharmaceutics and Biopharmaceutics 69 (2008) 1.

[15] H.S. Choi, W. Liu, P. Misra, E. Tanaka, J.P. Zimmer, I.B. Itty, M.G. Bawendi, J.V. Frangioni, Nature Biotechnology 25 (2007) 1165

[16] C. Gonçalves, J.A. Martins, F.M. Gama, Biomacromolecules 8 (2007) 392.

[17] C. Gonçalves, F.M. Gama, European Polymer Journal 44 (2008) 3529.

[18] E. Torrado, S. Adusumilli, A.G. Fraga, P.L.C. Small, A.G. Castro, J. Pedrosa, Infection and Immunity 75 (2007) 3979.

[19] J. Pedrosa, M. Florido, Z.M. Kunze, A.G. Castro, F. Portaels, J. McFadden, M.T. Silva, R. Appelberg, Clinical \& Experimental Immunology 98 (1994) 210.

[20] M.K. Warren, S.N. Vogel, The Journal Immunology 134 (1985) 982.

[21] R.J. Tushinski, I.T. Oliver, L.J. Guilbert, P.W. Tynan, J.R. Warner, E.R. Stanley, Cell 28 (1982) 71.

[22] X. Zhang, R. Goncalves, D.M. Mosser, Current Protocols in Immunology 83 (2008), 141.1-114.1.

[23] T. Mosmann, Journal of Immunological Methods 65 (1983) 55.
[24] L.C. Green, D.A. Wagner, J. Glogowski, P.L. Skipper, J.S. Wishnok, S.R. Tannenbaum, Analytical Biochemistry 126 (1982) 131.

[25] D. Hreczuk-Hirst, D. Chicco, L. German, R. Duncan, International Journal of Pharmaceutics 230 (2001) 57.

[26] B. Treetharnmathurot, L. Dieudonné, E. Ferguson, D. Schmaljohann, R. Duncan, R. Wiwattanapatapee, International Journal of Pharmaceutics 373 (2009) 68.

[27] C. Bocca, O. Caputo, R. Cavalli, L. Gabriel, A. Miglietta, M.R. Gasco, International Journal of Pharmaceutics 175 (1998) 185.

[28] C. Fang, B. Shi, Y. Pei, M. Hong, J. Wu, H. Chen, European Journal of Pharmaceutical Sciences 27 (2006) 27.

[29] A.N. Lukyanov, Z. Gao, L. Mazzola, V.P. Torchilin, Pharmaceutical Research 19 (2002) 1424.

[30] C.J. Rijcken, C.J. Snel, R.M. Schiffelers, C.F. Nostrum, W.E. Hennink, Biomaterials 28 (2007) 5581. 